\title{
Bending the Cost Curve and Improving Quality of Care in America's Poorest City
}

\author{
Andrea Miller, MA, Megan Cunningham, ${ }^{\star}$ and Nadia Ali, MPA
}

\begin{abstract}
In Camden, New Jersey-as in many urban areas-health care costs are concentrated in a disproportionately small number of patients. These "super-utilizers" typically have multiple chronic conditions combined with social barriers that make it hard to access and coordinate the care they need to manage these conditions and stay healthy. Launched in 2002, the Camden Coalition of Healthcare Providers strives to reform the quality, capacity, and accessibility of the health care system for the city's vulnerable populations. (Population Health Management 2013;16:S-17-S-19)
\end{abstract}

\section{Introduction}

I N CAMDEN, New JeRSEY - as in many urban areas-health care costs are concentrated in a disproportionately small number of patients. These "super-utilizers" typically have multiple chronic conditions combined with social barriers that make it hard to access and coordinate the care they need to manage these conditions and stay healthy. Launched in 2002, the Camden Coalition of Healthcare Providers (Coalition) strives to reform the quality, capacity, and accessibility of the health care system for the city's vulnerable populations.

Built on a partnership between providers, patients, and community resources, the Coalition includes active participation from all 3 Camden hospitals, 2 Federally Qualified Health Centers, private community-based medical practices, and social service agencies. By intervening with superutilizers and helping to reduce their hospital admissions, the Coalition works toward a dual goal of improving care for these patients while dramatically bending the cost curve. Because just $1 \%$ of the patients in Camden are responsible for $30 \%$ of health care spending, targeting these patients for a data-driven intervention significantly reduces costs. The Coalition also has been the driving force behind new state legislation that will allow for the creation of Medicaid Accountable Care Organizations in New Jersey.

\section{Ten Years of Improving Care Coordination}

The Coalition began in 2002 as an inspired idea in need of a concrete model. Initially, the program was an outreach agency working to sustain primary care in the city of Camden. Like many urban environments, Camden did not lack resources; rather, a guiding hand was needed to enable effective coordination.

With early funding support from the Robert Wood Johnson Foundation, the Coalition set to work building coalitions, looking beyond the work of primary care practices in pursuit of long-term impact. As this process continued and understanding deepened, the organization came to realize the critical nature of the social factors that dramatically influence patient wellness and recovery.

With further funding from Robert Wood Johnson in 2007, the Coalition expanded its scope to include a programbased approach that engaged housing agencies, homeless shelters, nutrition programs, and mental, behavioral, and homeless outreach. In 2009, the Coalition addressed the need for a clinical redesign and outreach effort. At the practice level, this entailed assessing continuity of care. The following year, the organization began to focus on patientlevel engagement.

Today, the sum of the Coalition's experience enables the organization to pursue its mission through legislation that improves care at the most fundamental and comprehensive levels. The Coalition is funded by foundation and federal grants in combination with contributions from members. It is governed by an executive board of directors comprised of representatives from Camden-area health care providers and social service organizations.

\section{A Framework for Change}

The Coalition has created a fully integrated care model in Camden. The organization's theory of change is to engage the entire provider community in clinical redesign activities 
that are informed by data analysis and that result in better care at lower costs. To this end, the Coalition focuses its efforts on:

- Clinical Redesign: Enhancing primary care through care coordination for medically and socially complex patients, peer education, support groups, and practice transformation activities at clinics across Camden.

- Community Engagement: Working closely with its member organizations and Camden residents to identify common goals and ensure broad participation in community-wide interventions.

- Data: Maintaining 3 significant data holdings that support its programs and initiatives:

○ A citywide health database that contains claims data from the 3 Camden hospitals to support populationlevel analyses of emergency department (ED) and inpatient utilization.

- The Camden Health Information Exchange (HIE) that allows providers to access detailed clinical data about their patients at the point of care.

- A customized care coordination tracking tool that is used to monitor and evaluate Coalition interventions.

\section{Link2Care: The Coalition's Care Management Intervention}

Beginning in July 2012, a Center for Medicare and Medicaid Innovations Healthcare Innovation Award provided funding for the Coalition to expand its outreach services to 870 frequent users of hospital and ED resources. Link2Care seeks to save $\$ 6$ million in health care costs over 3 years by reducing hospital readmissions and increasing access to primary and specialty care.

The Coalition's care management intervention starts with a daily feed from the HIE. This database provides a list of patients currently admitted to the 3 Camden hospitals represented in the HIE who have already been admitted twice in the past 6 months. From this list, patients are identified for intervention based on a qualitative checklist.

If a patient is identified for a care management intervention, a registered nurse (RN) staff member visits him or her at the bedside in the hospital. The RN asks the patient to describe his or her health situation and goals, particularly whether he or she would like help avoiding future hospitalizations. If the patient agrees to participate, the RN uses a risk stratification form to assess patient factors that may contribute to future readmission.

Once the patient is discharged from the hospital, a team comprised of an RN, a licensed practical nurse, and a social worker visits the patient at home. During this visit, the team helps the patient complete an extensive intake form that is used to help build a care plan, and assists the patient with making an appointment with his or her primary care physician (PCP). The goal is a follow-up appointment within 7 days along with any required specialist visits. The team performs medication reconciliation, comparing the prescriptions the patient has at home with the discharge summary from the hospital. A critical part of this initial home visit is setting goals based on the patient's needs and desired outcomes, as well as the steps needed to medically and socially stabilize the patient to prevent further readmissions.
A member of the care team accompanies the patient to the follow-up PCP visit as well as other necessary appointments. At this initial appointment, the RN reviews the patient's discharge summary, problem list, medication reconciliation, and symptom tracker with the PCP. The goal of this visit is to provide patient support while coordinating with the PCP in a partnership to help provide better care.

Weekly home visits continue for 30 days, at which point the team assesses whether the patient is medically and socially stable and able to manage his or her own care with the help of a PCP. Based on this assessment, the team makes one of the following determinations:

- The patient is ready to be graduated with a "warm handoff" to his or her PCP.

- The patient needs an additional 30-day intervention to complete the care plan.

- An extended engagement of more than 60 days is necessary for the patient to become medically and socially stable.

\section{A Data-Driven Initiative}

The Coalition's data initiative is supported primarily by the above-mentioned HIE, which offers Camden city and regional health care providers real-time access to patients' important medical information. This resource makes it possible to perform accurate predictive analyses-a step that is the heart of the Coalition's model-enabling better, more cost-effective care, reducing unnecessary duplication and inefficiencies, and fostering improved treatment coordination.

Innovative use of this data drives the patient engagement cycle. Used correctly, data can uncover system deficiencies and inform disease-specific metrics to identify high-utilizing patients.

Once the patient intervention is completed, data are an effective means to continually monitor his or her progress. Although qualitative feedback from patients is valuable, the numbers are critical to assess the population's health and identify gaps in the care delivery system.

In the long term, data make it possible to track patient utilization and gauge improvement. An individual may graduate from the Coalition's intervention program in 30 to 90 days, but his or her progress is monitored for up to 1 year to confirm sustained improvement.

\section{The Importance of Engagement}

The Coalition's intervention places nurses and health coaches in meaningful contact with both health care providers and patients in acute situations. Lasting improvement in care coordination and quality requires both parties to reimagine their established roles. Thus, Coalition staff play a critical role in empowering patients to more effectively advocate on their own behalf and proactively seek preventive care with their PCPs rather than relying on an ED visit. Physicians, meanwhile, benefit from the opportunity to view complex patients in new ways.

Beyond the care management intervention, the Coalition is working with several primary care practices in Camden to reengineer the care delivery system for complex patients. These initiatives embed nurse practitioners in small 
offices within high-cost centers to coordinate home-based nurse care.

\section{Additional Initiatives}

\section{Behavioral Health}

The Coalition's Behavioral Health (BH) Department is focused on providing and making accessible $\mathrm{BH}$ services for both its patients and the broader Camden community. The scope of work includes $\mathrm{BH}$ consultation and training for Coalition clinical program staff, direct client counseling services, integration of $\mathrm{BH}$ and primary care services, and improved relationships within the community.

\section{Cross-site learning}

The Cross-Site Learning Program uses a community-based approach to provide technical assistance for those working with high-utilizing patients in order to cut costs and improve health care quality and coordination. The technical assistance program includes case conferencing, individualized consultation, site visits, and topical webinars. The ultimate goal at the end of the 2-year grant cycle is for the sites to develop successful, scalable pilot programs and to serve as regional centers of excellence and teaching for super-utilizer work.

\section{Camden GPS}

The Camden GPS Program is a hospital-based intervention for adolescents and young adults aged 10-24 who have been victims of assault violence. Through individualized outreach services, such as case management, family support, and connections to community resources, GPS strives to strengthen family bonds while providing resources to deal with violence.

\section{Hotspotting fellowship}

The Fellowship Project aims to reduce the rate of hospital readmissions and ED utilization among Abigail House Rehabilitation Center patients by training PCPs to develop and implement strategies that address the issue of frequent utilization. Two family medicine physician fellows have been working closely with Abigail House, which serves many of the most complex patients in Camden, to evaluate its current health care operations and identify ways in which Abigail House and local hospitals can better coordinate care, particularly during the patient transfer process.

\section{Integrated Diabetes Care Program (IDC)}

IDC is a multidisciplinary effort to improve diabetes care at the patient, practice, and community levels. Broadly, the objectives of IDC are to build the capacity of primary care practices to provide innovative and comprehensive care to patients with diabetes; increase access to diabetes selfmanagement education; and improve the coordination of care for patients across health care providers, hospitals and health systems in Camden.

\section{New Jersey Medication Access Partnership (NJ MAP)}

NJ MAP ensures that patients with limited incomes have access to the prescription medications and education they need to control their chronic conditions. NJ MAP connects qualified patients who cannot afford their prescribed medications to programs run by various pharmaceutical companies to obtain the medications at low cost or no cost. The program operates out of 3 primary care sites in Camden.

\section{Pregnancy and Parenting Partners (P3)}

P3 combines group prenatal and well-child visits with education and support to address the reduced health and social functioning of high-risk and socially disadvantaged parents and their children. P3 consists of sessions with approximately 10 women of similar gestational ages, beginning in pregnancy and providing care through delivery and the infant's first year of life. Sessions are held every 2 weeks for 18 months. Benefits of the model include care from a physician, nurse, and other providers if needed, as well as a social support component.

\section{Looking Ahead}

The interplay of all these programs and initiatives is vital to the organization's mission of improving health care access for city residents while lowering costs. Community care outreach-actively changing the way practices work through sustained patient and practice engagement-is just as important as bending the cost curve. Put simply, change at this level challenges the broken but deeply entrenched status quo. As the national landscape of health care changes, the Coalition will continue to take active steps to improve the state of health care in Camden and to develop the ways these strategies are pursued, both as an individual organization and as part of a Coalition.

Address correspondence to: Andrea Miller, MA Camden Coalition of Healthcare Providers 800 Cooper Street, $7^{\text {th }}$ floor Camden, NJ 08102

E-mail: andrea@camdenhealth.org 